\title{
Avaliação da qualidade da Atenção Primária à Saúde segundo homens de um município mineiro
}

\author{
Quality evaluation of Primary Health Care according to men in a municipality in Minas Gerais \\ Evaluación de la calidad de la Atención Primaria de Salud según hombres en una ciudad de Minas \\ Gerais
}

Recebido: 03/05/2021 | Revisado: 11/05/2021 | Aceito: 14/05/2021 | Publicado: 30/05/2021

Gabriel dos Reis Pinto

ORCID: https://orcid.org/0000-0002-4794-1110 Universidade Federal de Alfenas, Brasil E-mail: gabrieldosreis16@gmail.com

Thaynara Macedo de Oliveira

ORCID: https://orcid.org/0000-0002-0935-1224

Universidade Federal de Alfenas, Brasil

E-mail: thaynaramacedo14@gmail.com

Renata Messias Frazão

ORCID: https://orcid.org/0000-0002-9430-0500 Universidade Federal de Alfenas, Brasil

E-mail: renatamfrazao@gmail.com

Ritiellen Amanda Ferreira dos Santos

ORCID: https://orcid.org/0000-0002-9523-6387 Universidade Federal de Alfenas, Brasil

E-mail: ritiellen.afs@gmail.com

Denismar Alves Nogueira

ORCID: https://orcid.org/0000-0003-2285-8764 Universidade Federal de Alfenas, Brasil

E-mail: denismar.nogueira@unifal-mg.edu.br

Simone Albino da Silva

ORCID: https://orcid.org/0000-0002-2725-8832 Universidade Federal de Alfenas, Brasil E-mail: simone.silva@unifal-mg.edu.br Murilo César do Nascimento

ORCID: https://orcid.org/0000-0002-3436-2654 Universidade Federal de Alfenas, Brasil

E-mail: murilo@unifal-mg.edu.br

\begin{abstract}
Resumo
O objetivo foi de descrever a qualidade da Atenção Primária à Saúde (APS) segundo usuários do sexo masculino. Pesquisa avaliativa, transversal, em que foram entrevistados 228 homens em domicílio, de fevereiro a julho de 2019 , em áreas de oito Estratégias de Saúde da Família de um município mineiro. Utilizaram-se os recursos Google Forms (versão reduzida do PCATool), Excel, SPSS e R. Adotaram-se os testes qui-quadrado de Pearson, Exato de Fisher e de Mann-Whitney, a um nível de significância de 5\%. Os participantes perfilaram como idosos, pardos, nascidos noutras cidades, que moravam com mais uma pessoa, casados, instruídos da $1^{\text {a }}$ a $4^{\text {a }}$ série do ensino fundamental, aposentados e com renda de 1,5 até 3 salários mínimos. Destacaram-se autorrelatos de hipertensão arterial, diabetes, uso de álcool, doença renal e problema de saúde mental. Dentre os entrevistados, 80,70\% avaliaram a atenção primária como de baixa qualidade. Houve associação entre ter hipertensão arterial sistêmica e a qualidade da APS ( $\mathrm{p}=$ 0,001). Assim, foram contemplados os objetivos do estudo, ressaltando-se a má qualidade da APS na visão de homens pertencentes a grupos de vulnerabilidade social e a necessidade da ampliação do acesso do homem jovem à atenção primária.
\end{abstract}

Palavras-chave: Saúde do homem; Vulnerabilidade em saúde; Atenção primária à saúde; Qualidade, acesso e avaliação da assistência à saúde.

\begin{abstract}
The objective was to describe the quality of the primary health care (PHC) according to male users. This is an evaluative and cross-sectional study in which 228 men were interviewed at home, from February to July of 2019, in areas of eight Family Health Strategies in a municipality in Minas Gerais. The resources Google Forms (reduced version of PCATool), Excel, SPSS and R were used. Pearson's chi-square, Fisher's Exact and Mann-Whitney tests were used, at a significance level of 5\%. The participants profiled as elderly, mixed race, born in other cities, who
\end{abstract}


lived with one more person, married, educated from the first to the fourth grade of elementary school, retired and with income from 1.5 up to 3 minimum wages. Self-reports of hypertension, diabetes, alcohol use, kidney disease and mental health problem were highlighted. Among the interviewees, $80.70 \%$ rated the PHC as of low quality. There was association between having hypertension and the quality of the PHC $(\mathrm{p}=0.001)$. Thus, the objectives of this study were achieved, emphasizing the poor quality of PHC in the view of men belonging to socially vulnerable groups and the need to expand the access of young men to the PHC.

Keywords: Men's health; Health vulnerability; Primary health care; Health care quality, access, and evaluation.

\section{Resumen}

El objetivo fue describir la calidad de la Atención Primaria de Salud (APS) según usuarios del sexo masculino. Encuesta evaluativa, transversal, en la que se entrevistó a 228 hombres a domicilio, de febrero a julio de 2019, en áreas de ocho Estrategias de Salud de la Familia en una ciudad de Minas Gerais. Se utilizaron los recursos de Google Forms (versión reducida del PCATool), Excel, SPSS y R. Se adoptaron las pruebas chi-cuadrado de Pearson, Exacto de Fisher y Mann-Whitney, con un nivel de significación de 5\%. Los participantes fueron perfilados como ancianos, marrón, nacidos en otras ciudades, que convivían con una persona más, casados, educados de la $1^{\circ}$ al $4^{\circ}$ años de la primaria, jubilados y con ingresos mensuales de 1,5 hasta 3 salarios mínimos. Se destacaron los autoinformes de hipertensión arterial, del diabetes, consumo de alcohol, enfermedades renales y problemas de salud mental. Entre los entrevistados, el 80,70\% evaluó la atención primaria como de baja calidad. Hubo asociación entre tener hipertensión arterial sistémica y la calidad de la APS $(\mathrm{p}=0.001)$. Así, se contemplaron los objetivos del estudio, destacando la mala calidad de la APS en la visión de los hombres pertenecientes a grupos socialmente vulnerables y la necesidad de ampliar el acceso de los hombres jóvenes a la atención primaria.

Palabras clave: Salud del hombre; Vulnerabilidad en salud; Atención primaria de salud; Calidad, acceso y evaluación de la atención de salud.

\section{Introdução}

No Brasil, a Atenção Primária à Saúde é oficialmente chamada de Atenção Básica, sendo regulamentada por uma portaria que traz como cerne que esta é a porta principal do sistema de saúde brasileiro e implantada preferencialmente pela Estratégia da Saúde da Família - ESF (Brasil, 2017). Abarca um conjunto de ações de saúde que visam à integralidade do cuidado à pessoa humana de forma individual, familiar e coletiva; e tem potencial para alcançar impactos positivos quando contempla os atributos de acesso de primeiro contato, longitudinalidade, integralidade e coordenação da atenção, atenção à saúde centrada na família, orientação comunitária e competência cultural (Brasil, 2017; Starfield, 2002).

A organização do processo de trabalho das equipes da ESF permite a aplicabilidade desses atributos, de modo que as necessidades em saúde da população sejam contempladas pelo serviço, criando condições para uma abordagem que transcenda o foco patológico (Starfield, 2002).

Nesse contexto organizacional da saúde, foi publicada em 2009 a Política Nacional de Atenção Integral à Saúde do Homem (PNAISH), destinada ao homem brasileiro na faixa etária entre 20 a 59 anos, visando desenvolver ações que diminuam a morbimortalidade, incentivem o autocuidado não se restringindo às práticas pontuais, como ações de prevenção do câncer de próstata (Brasil, 2009; Bidinotto, Simonetti \& Bocchi, 2016).

Há baixa presença masculina nos serviços de atenção básica, tendo esta a ligação com o fator social de que os são conferidos o papel laboral e a responsabilidade pela renda familiar, o grande tempo de espera para o atendimento e a baixa resolutividade do serviço (Ribeiro, Gomes \& Moreira, 2017). Destaca-se, ainda, que a construção social de que o homem não deve mostrar suas fragilidades, de que não aceita a possibilidade de ficar doente, justificando um menor cuidado com a própria saúde e refletindo negativamente na realização de práticas de prevenção e na aceitação de tratamento médico (Queiroz et al., 2018; Portela et al., 2016).

Tendo em vista que os atributos da APS têm potencial para direcionar o cuidado à saúde, e que a pesquisa avaliativa na realidade do serviço tem como característica pontuar carências e fragilidades do objeto sob investigação, produzindo de insumos diretivos para a mudança, melhoria da prática assistencial e melhoria da qualidade (Salci, Silva \& Meirelles, 2018), buscou-se responder a seguinte questão: "Como os adultos do sexo masculino atendidos pela Estratégia de Saúde da Família de 
um município mineiro avaliam a qualidade da APS?". Além disso, este estudo também objetivou realizar uma caracterização sociodemográfica e das condições/situações de saúde desses usuários adultos do sexo masculino, verificando se existe associação entre as variáveis sociodemográficas e das condições/situações de saúde dos usuários entrevistados com os escores de qualidade da APS por eles gerados.

\section{Metodologia}

\section{Tipo, local e período de estudo}

Trata-se de uma pesquisa avaliativa, transversal (descritiva-analítica) na qual foram entrevistados homens assistidos por oito equipes de ESF em um município mineiro. A coleta de dados ocorreu por meio de entrevistas no período de 20 de fevereiro de 2019 a 15 de julho de 2019.

\section{Participantes do estudo, critérios de seleção}

Os participantes do estudo foram selecionados por meio de uma amostragem por conveniência. Os Agentes Comunitários de Saúde (ACS) das unidades abordadas indicaram indivíduos pertencentes a sua microárea de atuação que preenchiam os critérios de seleção: ser do sexo masculino, maior de 18 anos e estar disponível para a entrevista domiciliar no período das 8 horas às 17 horas. Os dados eram disponibilizados por meio de uma lista contendo nome e endereço sendo que se procurou dividir os participantes de modo equânime entre as microáreas. Ademais, buscou-se obter um número idêntico de entrevistados entre as unidades de saúde, de modo que ao final dos 4 meses e 25 dias de pesquisa, o estudo contou com 228 entrevistados. Assim, a escolha do método de amostragem se apresentou como uma limitação do estudo, porém com a manutenção do potencial de abrangência dos resultados a nível municipal da cidade mineira participante.

\section{Aspectos éticos}

O estudo foi aprovado pelo Comitê de Ética em Pesquisa da instituição proponente e contou com a autorização pela Secretaria Municipal de Saúde do município mineiro estudado (CAAE $n^{\circ}$ 97909018.0.0000.5142 e parecer de $\mathrm{n}^{\circ}$ 3.695.990). Os convidados participaram da entrevista após a aceitação dos aspectos contidos no Termo de Consentimento Livre e Esclarecido (TCLE).

\section{Protocolo da coleta de dados}

As entrevistas foram realizadas exclusivamente em domicílio. O questionário foi composto por três seções: identificação sociodemográfica, condições de saúde autorreferidas e Instrumento de Avaliação da Atenção Primária, o Primary Care Assessment Tool (PCATool) versão adulto reduzida, que avalia a qualidade da APS por meio dos atributos essenciais e derivativos (Oliveira et al., 2013).

O PCATool é um instrumento de fácil aplicabilidade, sendo baseado no modelo de avaliação de Donabedian e na tríade por ele destacada (estrutura, processo e resultado), apresentando inúmeras versões que permitem avaliar a APS a partir do ponto de vista dos profissionais, usuários adultos e, até mesmo, das crianças (Paula et al., 2016). É amplamente utilizado tanto como ferramenta de pesquisa acadêmica quanto para monitoramento por órgãos gestores, ou até mesmo pela própria Equipe de Saúde da Família, apresentando potencial para contribuir com melhorias na qualidade da APS (Brasil, 2010).

O instrumento conta com 25 itens, sendo que 22 deles que permitem como possibilidades de respostas: "com certeza sim", "provavelmente sim", "provavelmente não", "com certeza não" e "não sei/não lembro" que correspondem, respectivamente, aos valores 4, 3, 2, 1 e 2, exceto para as questões com código C11 e D15, em que quanto maior o valor conferido pelo participante nas opções de resposta, menor é a qualidade da avaliação; frente a isso, seus valores são invertidos 
para o cálculo do escore - valor 4 equivale a 1; valor 3 equivale a 2; valor 2 equivale a 3; valor 4 equivale a 1. Três itens são compostos por uma pergunta cada referente à afiliação, permitindo "sim" e "não" como opções de resposta, sendo que caso todas as respostas sejam negativas, o valor será 1; a presença de uma ou mais respostas positivas referentes a serviços diferentes fornecem valor 2; duas respostas positivas referentes a um mesmo serviço geram o valor 3; e todas as respostas positivas propiciam o valor 4 (Brasil, 2010).

O escore de um componente foi obtido pela média aritmética simples dos valores dos itens que o compõe, enquanto a média de todos os componentes forneceu o escore geral (Brasil, 2010), sendo, posteriormente, calculado o escore padronizado, gerando uma escala que varia de 0 a 10, de modo que valores iguais ou maiores que 6,6 representam um alto escore, indicando o alcance de boa qualidade na APS. O cálculo do escore padronizado apresenta como base o escore geral, sendo realizado pela fórmula (Oliveira, 2007):

\section{Escore Padronizado $=\frac{(\text { Escore-1) }}{(4-1)} \times 10$}

A identificação sociodemográfica foi adaptada a partir da Ficha Individual e Domiciliar de Cadastro do Sistema de Informação em Saúde para a Atenção Básica (SISAB) do e-SUS, assim como o questionário das condições de saúde autorreferidas, composta por 23 perguntas (Brasil, 2018). Desta forma, a pesquisa apresentou como variáveis independentes as características sociodemográficas e as condições autorreferidas e como variável dependente o escore geral. O formulário foi delineado para ser aplicado através da ferramenta eletrônica Google Forms. Entretanto, devido à instabilidade da rede de internet, em duas entrevistas foi necessária a utilização do formulário em sua forma impressa sendo, os dados posteriormente lançados pelos pesquisadores no formulário eletrônico.

\section{Análise dos resultados e estatística}

A tabulação das respostas se deu utilizando a ferramenta Microsoft Excel por dupla entrada e suas análises foram realizadas, majoritariamente, no Statistical Package for the Social Sciences (SPSS), utilizando, a priori, o qui-quadrado de Pearson para análise da associação entre as variáveis dependentes (escores) e independentes (caracterização socioeconômica e condições de saúde autorreferidas) com posterior análise descritiva. Entretanto, este teste pode comprometer a análise dos dados quando não há um mínimo de cinco respostas em cada célula disponível; portanto, a fim de se obter uma melhor análise dos dados, frente a esses casos, foram utilizados o teste exato de Fisher, para matrizes 2x2, e o software $\mathrm{R}$ para matrizes maiores que 2x2. O nível de significância adotado para os testes foi de 5\% e o intervalo de confiança de $95 \%$.

\section{Resultados}

Ao final da coleta de dados, 422 homens foram selecionados para o estudo, sendo que 172 destes não se encontravam no momento da entrevista. Dos 250 que se apresentavam em ambiente domiciliar, após a apresentação dos pontos contemplados pelo TCLE, 22 se recusaram a participar da entrevista e 228 aceitaram realizá-la. Dos entrevistados, a maior parcela foi composta por indivíduos com 60 anos ou mais ( $\mathrm{n}=142$ / 62,28\%) e autodeclarados pardos ( $\mathrm{n}=106 / 46,49 \%)$, seguidos pela identificação como branco $(\mathrm{n}=92 / 40,35 \%)$, preto $(\mathrm{n}=29 / 12,72 \%)$ e amarelo $(\mathrm{n}=1 / 0,44 \%)$. Quanto à naturalidade, $36,84 \%(n=84)$ nasceram em Alfenas; 46,49\% $(n=106)$ nas demais cidades mineiras e 16,67\% $(n=38)$ em municípios de outros estados.

Houve um predomínio de homens que dividem a residência com apenas uma pessoa ( $\mathrm{n}=74$ / 32,46\%), sucedidos por aqueles que vivem com: mais duas pessoas ( $\mathrm{n}=61 / 26,75 \%)$; sozinhos $(\mathrm{n}=30 / 13,16 \%)$; mais três $(\mathrm{n}=29 / 12,72 \%)$, quatro 
( $n=15 / 6,58 \%)$, cinco $(n=9 / 3,95 \%)$, seis $(n=8 / 3,51 \%)$ ou mais $(n=2 / 0,88 \%)$ indivíduos. No que se refere ao estado civil, no momento da entrevista, 58,33\% $(\mathrm{n}=133)$ eram casados, $19,74 \%(\mathrm{n}=45)$ solteiros, 10,96\% $(\mathrm{n}=25)$ divorciados, 10,53\% $(\mathrm{n}=24)$ viúvos e um entrevistado $(0,44 \%)$ se enquadrou na opção "outros". Em relação à escolaridade, 46,93\% (n=107) dos entrevistados estudaram entre a $1^{\mathrm{a}}$ e $4^{\mathrm{a}}$ série do ensino fundamental, $14,47 \% \quad(\mathrm{n}=33)$ cursaram da $5^{\mathrm{a}}$ à $8^{\mathrm{a}}$ série da mesma modalidade e 4,36\% ( $n=10)$ completaram esse módulo. O Ensino Médio foi frequentado por 13,60\% ( $n=31)$ dos entrevistados, enquanto $0,88 \%(\mathrm{n}=2)$ estudaram por meio da Educação para Jovens e Adultos (EJA). A porcentagem de indivíduos que nunca frequentaram a escola $(\mathrm{n}=27 / 11,84 \%)$ mostrou-se superior à porcentagem dos que apresentavam ensino superior completo $(\mathrm{n}=16 / 7,02 \%)$, frequentaram a pré-escola $(\mathrm{n}=1 / 0,44 \%)$ ou classe de alfabetização $(\mathrm{n}=1 / 0,44 \%)$.

Houve um predomínio de aposentados $(\mathrm{n}=160 / 70,18 \%)$, seguidos por autônomos sem previdência social ( $\mathrm{n}=18 /$ 7,89\%), desempregados ( $\mathrm{n}=17 / 7,46 \%)$, assalariados com carteira de trabalho ( $\mathrm{n}=14$ / 6,14\%), autônomos com previdência social $(\mathrm{n}=10 / 4,39 \%)$, assalariados sem carteira de trabalho $(\mathrm{n}=5 / 2,19 \%)$, indivíduos que não trabalham $(\mathrm{n}=2 / 0,88 \%)$, servidores públicos e militares ( $\mathrm{n}=1 / 0,44 \%)$ e outros $(\mathrm{n}=1 / 0,44 \%)$. Por fim, quanto à renda familiar mensal, 46,05\% $(\mathrm{n}=105)$ dos núcleos familiares recebem de 1,5 até 3 salários mínimos por mês, enquanto 17,54\% (n=40) recebem acima de 3 a 4,5 salários mínimos; $16,67 \%(\mathrm{n}=38)$ recebem acima de 1 a 1,5 salários mínimos; 14,04\% (n=32) recebem até um salário mínimo; $3,07 \%(n=7)$ recebem acima de 4,5 até 6 salários mínimos e 2,63\% (n=6) recebem acima de 6 a 10 salários mínimos, sendo que nenhum entrevistado apresentava renda familiar superior a 10 salários mínimos.

Já em relação ao instrumento PCATool, para o grau de afiliação, 125 entrevistados $(54,82 \%)$ denotaram valor 4; 51 $(22,36 \%)$ valor $3 ; 52(22,80 \%)$ valor 2 ; e $0(0 \%)$ valor 1 ; sendo que quanto maior o valor gerado, melhor é a avaliação para esse componente. Ademais, os outros resultados obtidos referentes ao serviço de saúde ofertados pela ESF de cobertura dos entrevistados se encontram na Tabela 1. As condições de saúde autorreferidas, por sua vez, apresentavam "sim" e "não" como possibilidades de respostas, de modo que se obteve as relações de frequência por condição contidas no Gráfico 1.

Acrescentou-se ao banco de dados um campo denominado qualidade da APS, sendo uma variável dicotômica qualitativa, em que as escalas padronizadas maiores ou iguais a 6,6 receberam o número 1, indicando uma APS de alta qualidade, e valores inferiores a 6,6 pontuaram 2, denotando uma APS de baixa qualidade (Oliveira, 2007). Dessa forma, 44 entrevistas pontuaram $\geq 6,6$ e 184 entrevistas obtiveram $<6,6$, sendo que as frequências relativas resultantes de tal análise estão compreendidas no Gráfico 2. 
Tabela 1 - Frequência absoluta e relativa dos itens referentes aos atributos do instrumento PCATool-Adulto-Brasil versão reduzida sob perspectiva de homens assistidos por Estratégias de Saúde da Família de um município mineiro. Alfenas-MG, 2019.

\begin{tabular}{|c|c|c|c|c|c|c|c|c|c|}
\hline \multirow{3}{*}{ Atributos } & \multirow{3}{*}{$\begin{array}{l}\text { Perguntas } \\
\text { / Códigos }\end{array}$} & \multicolumn{8}{|c|}{ Alternativas / Respostas } \\
\hline & & \multicolumn{2}{|c|}{$\begin{array}{c}\text { Com certeza } \\
\text { sim }\end{array}$} & \multicolumn{2}{|c|}{$\begin{array}{c}\text { Provavelmente } \\
\text { sim }\end{array}$} & \multicolumn{2}{|c|}{$\begin{array}{c}\text { Provavelmente } \\
\text { não } \\
\text { Não sei / não } \\
\text { lembro } \\
\end{array}$} & \multicolumn{2}{|c|}{$\begin{array}{c}\text { Com certeza } \\
\text { não }\end{array}$} \\
\hline & & $\mathbf{N}$ & $\%$ & $\mathbf{N}$ & $\%$ & $\mathbf{N}$ & $\%$ & $\mathbf{N}$ & $\%$ \\
\hline $\begin{array}{c}\text { Primeiro contato } \\
\text { (Utilização) }\end{array}$ & $\mathrm{B} 2$ & 98 & 42,98 & 78 & 34,21 & 40 & 17,54 & 12 & 5,26 \\
\hline \multirow{2}{*}{$\begin{array}{l}\text { Primeiro contato } \\
\text { (Acesso) }\end{array}$} & $\mathrm{C} 4$ & 34 & 14,91 & 5 & 2,19 & 171 & 75,00 & 18 & 7,89 \\
\hline & $\mathrm{C} 11$ & 56 & 24,56 & 37 & 16,23 & 52 & 22,81 & 83 & 36,40 \\
\hline \multirow{4}{*}{$\begin{array}{l}\text { Longitudinalidade } \\
\text { (Atendimento } \\
\text { continuado) }\end{array}$} & D1 & 63 & 27,63 & 43 & 18,86 & 57 & 25,00 & 65 & 28,51 \\
\hline & D6 & 206 & 90,35 & 13 & 5,70 & 6 & 2,63 & 3 & 1,32 \\
\hline & D9 & 95 & 41,67 & 71 & 31,14 & 36 & 15,79 & 26 & 11,40 \\
\hline & D15 & 30 & 13,16 & 18 & 7,89 & 38 & 16,67 & 142 & 62,28 \\
\hline \multirow{5}{*}{$\begin{array}{l}\text { Coordenação } \\
\text { (Cuidado e } \\
\text { Sistemas de } \\
\text { informação) }\end{array}$} & E6 & 133 & 58,33 & 7 & 3,07 & 22 & 9,65 & 66 & 28,95 \\
\hline & E10 & 89 & 39,04 & 23 & 10,09 & 62 & 27,19 & 54 & 23,68 \\
\hline & E11 & 58 & 25,44 & 17 & 7,46 & 63 & 27,63 & 90 & 39,47 \\
\hline & E13 & 25 & 10,96 & 14 & 6,14 & 64 & 28,07 & 125 & 54,82 \\
\hline & F3 & 187 & 82,02 & 14 & 6,14 & 24 & 10,53 & 3 & 1,32 \\
\hline \multirow{3}{*}{$\begin{array}{c}\text { Integralidade } \\
\text { (Serviços } \\
\text { Disponíveis) }\end{array}$} & G9 & 41 & 17,98 & 13 & 5,70 & 14 & 6,14 & 160 & 70,18 \\
\hline & G17 & 79 & 34,65 & 16 & 7,02 & 13 & 5,70 & 120 & 52,63 \\
\hline & $\mathrm{G} 20$ & 31 & 13,60 & 10 & 4,39 & 8 & 3,51 & 179 & 78,51 \\
\hline \multirow{4}{*}{$\begin{array}{c}\text { Integralidade } \\
\text { (Serviços } \\
\text { Prestados) }\end{array}$} & H1 & 124 & 54,39 & 16 & 7,02 & 6 & 2,63 & 82 & 35,96 \\
\hline & H5 & 140 & 61,40 & 15 & 6,58 & 5 & 2,19 & 68 & 29,82 \\
\hline & $\mathrm{H} 7$ & 100 & 43,86 & 27 & 11,84 & 28 & 12,28 & 73 & 32,02 \\
\hline & H11 & 58 & 25,44 & 10 & 4,39 & 11 & 4,82 & 149 & 65,35 \\
\hline \multirow{2}{*}{ Enfoque Familiar } & I1 & 27 & 11,84 & 31 & 13,60 & 33 & 14,47 & 137 & 60,09 \\
\hline & I3 & 35 & 15,35 & 68 & 29,82 & 87 & 38,16 & 38 & 16,67 \\
\hline $\begin{array}{c}\text { Orientação } \\
\text { Comunitária }\end{array}$ & $\mathrm{J} 4$ & 14 & 6,14 & 19 & 8,33 & 19 & 8,33 & 176 & 77,19 \\
\hline
\end{tabular}

Legenda: B2: prioridade a ir nesse serviço frente a um novo problema de saúde; $\mathrm{C} 4$ : consegue atendimento rápido por telefone quando a UBS se encontra aberta; C11: existe dificuldade para conseguir atendimento médico; D1: atendimento pelo mesmo profissional; D6: sentir-se à vontade nas consultas; D9: serviço e profissionais de saúde sabem quais são os problemas mais importantes para você; D15: mudaria de serviço de saúde caso fosse fácil; E6: o médico(a) já sugeriu consulta com um especialista; E10: o médico(a) informou ao especialista o motivo da consulta; E11: o médico(a) sabe os resultados da consulta com o especialista; E13: o médico(a) se interessou sobre a qualidade do atendimento com o especialista; F3: disponibilidade dos prontuários nas consultas; G9: recebeu aconselhamento sobre problemas de saúde mental; G17: recebeu aconselhamento sobre como parar de fumar; G20: recebeu aconselhamento sobre as mudanças que ocorrem com o envelhecimento; H1: recebeu aconselhamento sobre alimentação saudável; H5: recebeu aconselhamento sobre exercícios físicos; H7: verificação dos medicamentos em uso; H11: recebeu aconselhamento sobre prevenção de quedas; I1: inclusão no planejamento do tratamento e cuidado individual ou familiar; I3: organização de reunião familiar, caso necessário; J4: participação em pesquisas com pacientes sobre a qualidade do serviço.

Fonte: Autores. 
Gráfico 1 - Condições de saúde autorreferidas por homens assistidos por Estratégias de Saúde da Família de um município mineiro. Alfenas-MG, 2019.

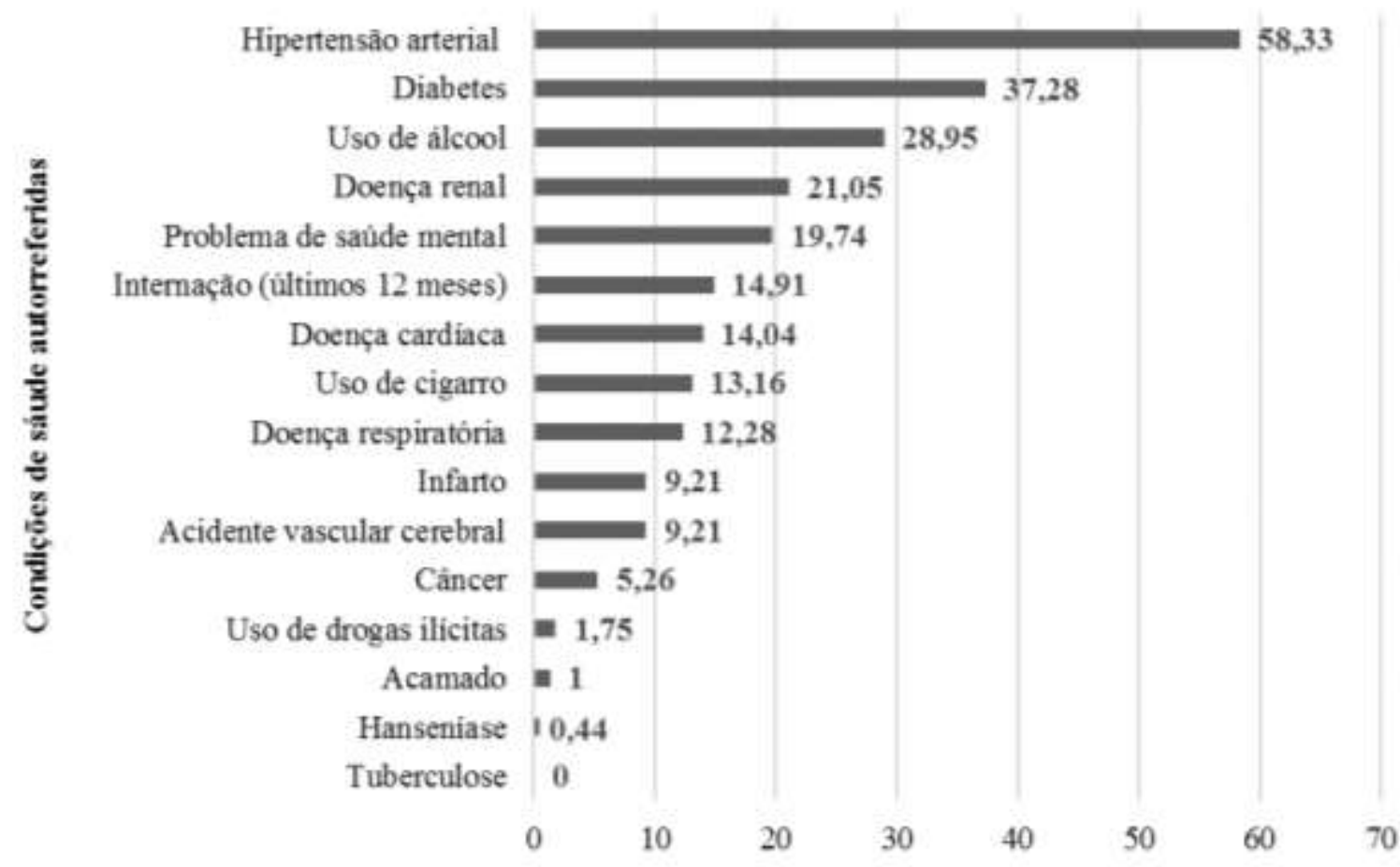

Frequência (\%)

Fonte: Autores.

Gráfico 2 - Frequência relativa da qualidade da Atenção Primária à Saúde obtida em entrevistas com homens assistidos por Estratégias da Saúde da Família de um município mineiro. Alfenas-MG, 2019.

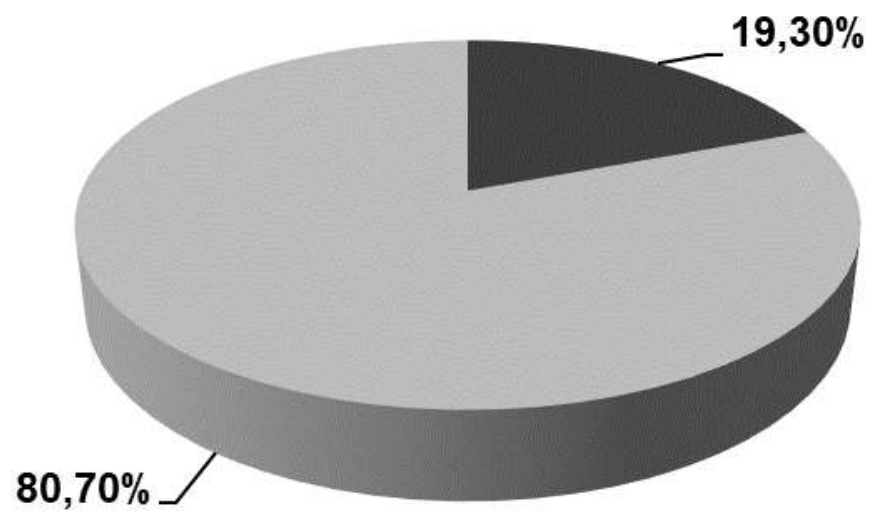

- Boa qualidade $\quad$ Má qualidade

Fonte: Autores.

Para verificar a presença de associação entre as variáveis independentes idade, número de pessoas da família que compartilham a mesma casa e renda familiar com a nova variável dependente binária criada (qualidade da APS), foi utilizado o teste não paramétrico de Mann-Whitney. A partir disso, não foi observada associação entre tais variáveis, ausência essa constatada por meio dos p valores iguais a $0,062,0,355$ e 0,186 , respectivamente.

Ao teste do qui-quadrado de Pearson, também não houve associação entre a variável dependente qualidade da APS e as variáveis independentes não binárias raça/cor $(\mathrm{p}=0,962)$, município e estado de nascimento $(\mathrm{p}=0,743)$, estado civil $(\mathrm{p}=$ 
0,292), curso mais elevado que frequenta ou frequentou $(\mathrm{p}=0,146)$, situação no mercado de trabalho $(\mathrm{p}=0,541)$, e autoconsideração sobre o peso $(\mathrm{p}=0,748)$.

Sobre a relação entre as variáveis binárias independentes e a variável dependente sobre a qualidade, foi encontrada associação entre ter hipertensão arterial e a qualidade da APS $(\mathrm{p}=0,001)$. Contudo, não houve relação entre a qualidade de APS e as respostas às perguntas: está fumante? $(\mathrm{p}=0,548)$; faz uso de álcool? $(\mathrm{p}=0,785)$; tem diabetes? $(\mathrm{p}=0,052)$; tem doença cardíaca/do coração? $(\mathrm{p}=0,570)$; tem ou teve problemas nos rins? $(\mathrm{p}=0,762)$; tem doença respiratória/no pulmão? (p = 0,760); teve alguma internação nos últimos 12 meses? $(\mathrm{p}=0,836)$; teve diagnóstico de algum problema de saúde mental por profissional de saúde? $(\mathrm{p}=0,069)$.

A ausência de associação também foi identificada como resultado do teste exato de Fisher para a variável dependente qualidade da APS e as variáveis independentes binárias: uso de outras drogas $(\mathrm{p}=0,579)$, AVC/derrame $(\mathrm{p}=0,567)$, infarto $(\mathrm{p}$ $=0,567)$, hanseníase $(p=1,000)$, câncer $(p=0,705)$, e estar acamado $(p=0,096)$.

\section{Discussão}

No que se refere à caracterização sociodemográfica dos entrevistados, observa-se uma predominância significativa de indivíduos com 60 anos ou mais, dado também encontrado na pesquisa de avaliação da qualidade da Atenção Primária à Saúde na visão masculina, realizada em Teresina, no Piauí (Silva et al., 2018). Ademais, houve um predomínio de aposentados, o que se deve, provavelmente, à facilidade de encontrar esse público em seu domicílio em horário comercial, assemelhando-se ao período de funcionamento das Unidades Básicas de Saúde. Assim, apesar do viés de estudo obtido, pode-se destacar a necessidade de implantação de táticas que contribuam para a acessibilidade dos homens jovens, tais como estender o funcionamento da UBS para o período noturno e/ou finais de semana (Silva et al., 2018).

Quanto à autodeclaração para raça/cor, a maioria dos entrevistados se autodeclararam como negros (somatório de pretos e pardos), o que vai ao encontro de um estudo realizado na capital mineira que apresenta que essa população é a que mais utiliza serviços do Sistema Único de Saúde (Lages et al., 2017). Ademais, em relação ao local de nascimento, observou-se uma predominância de homens oriundos de outras cidades mineiras, algo que corrobora para a ideia de que Alfenas tem funcionado como cidade atrativa nos últimos tempos (Moura \& Vale, 2019).

Uma pesquisa de base populacional realizada no Distrito Federal demonstrou que indivíduos com menor poder aquisitivo e escolaridade tendem a utilizar preferencialmente os serviços da APS (Poças et al., 2019), sendo dados concordantes com o presente estudo. Desta forma, considerando as fragilidades econômicas e educacionais da maior parcela dos entrevistados, nota-se a necessidade da adaptação dos profissionais de saúde à realidade sociocultural dessas pessoas, considerando, também, suas visões de mundo e compreensões com relação aos processos de saúde, doença e autocuidado. O sucesso terapêutico depende de uma boa relação médico-paciente que só será construída a partir da disposição dos profissionais em promover uma escuta ativa e de uma comunicação adequada, feita através de uma linguagem mais acessível, favorecendo uma maior participação do indivíduo no que tange aos cuidados com a sua saúde (Souza et al., 2020).

Frente aos resultados da análise estatística do estudo, pode-se destacar a associação entre usuários do sexo masculino que autorreferiram serem portadores de hipertensão arterial sistêmica (HAS) com uma maior qualidade conferida à Atenção Primária da área coberta pelas ESF participantes. A abordagem de uma doença crônica, como a HAS, na ESF, é longitudinal (Moura, Ribeiro \& Paes, 2019), sendo que o manejo da condição inclui consultas mensais, em que se realiza a aferição da pressão arterial sistêmica e das medidas antropométricas, além da oferta de orientações sobre alimentação saudável, prática de exercícios físicos e terapia medicamentosa (Dantas \& Roncalli, 2019), de modo que tais instruções apresentaram "com certeza sim" como resposta mais predominante no presente estudo. Um inquérito domiciliar demonstrou que a ESF foi capaz de diminuir desigualdades envolvidas na abordagem à saúde do hipertenso no Brasil, funcionando como um relevante atenuador 
da mortalidade relacionada com a condição (Oliveira et al., 2020). Desta forma, é possível inferir que os homens hipertensos do estudo avaliaram melhor a qualidade da atenção primária devido à importante atuação da Estratégia de Saúde da Família para com essa patologia.

Ainda no campo das doenças crônicas, diabetes mellitus foi a segunda condição com número mais expressivo, consoante com as informações apresentadas pela Sociedade Brasileira de Diabetes, no qual o Brasil foi colocado como o $4^{\circ}$ país no ranking mundial, havendo forte aumento da condição na população masculina, com mais de 65 anos e baixa escolaridade (Sociedade Brasileira de Diabetes, 2019). É importante destacar que os pontos envolvidos nessa patologia, relacionados tanto ao acometimento, quanto ao manejo na APS e seus respectivos resultados, permeiam questões de desigualdades, compromisso por parte do poder público e influências midiáticas, alimentícias e farmacêuticas (Borges \& Lacerda, 2018), demonstrando a complexidade da abordagem da doença.

Com isso, um estudo nacional evidenciou que apenas $8 \%$ das UBS brasileiras dispunham de todo material necessário para um manejo de qualidade do paciente diabético e que $40 \%$ apresentavam disponibilidade dos medicamentos utilizados na terapêutica da condição (Neves et al., 2018). Além das deficiências quanto a medicação e materiais, a literatura aponta a baixa adesão no treinamento de funcionários e as dificuldades quanto ao processo de referência e contrarreferência como pontos a serem melhorados para alcançar uma efetiva qualidade na abordagem do diabetes mellitus (Huque et al., 2018).

Dessa forma, é possível perceber a importância da Atenção Primária em ações articuladas em promoção, prevenção e reabilitação, evitando-se assim, a instalação de condições com posterior cronificação, de modo a afetar permanentemente a vida do indivíduo (Balbino et al., 2020). Em condições já cronificadas, por sua vez, tem-se a relevância da manutenção de programas para o acompanhamento dessas patologias visando auxiliar na prevenção de possíveis complicações e agravos, além de promover uma melhora na qualidade de vida. O manejo das condições crônicas na APS abrange, ainda, o campo financeiro, uma vez que estas, quando agudizadas, podem passar a exigir a utilização de tecnologias de alto custo, principalmente, do nível terciário (Oliveira, Veras \& Cordeiro, 2018).

Diretrizes da atenção à reabilitação da pessoa com acidente vascular cerebral apresenta alguns fatores de risco para essa condição, como idosos; sexo masculino; portador de HAS e diabetes mellitus; alcoolismo; tabagismo; sedentarismo (Brasil, 2013), sendo que uma parcela considerável de participantes do presente estudo apresenta tais características, que são passíveis de redução ou até mesmo eliminação no contexto de atuação da APS.

Apesar da maioria dos homens entrevistados já ter sido encaminhada alguma vez para o serviço especializado, foram, por várias vezes, relatadas esperas de anos para se ter acesso aos exames e ao serviço especializado por meio do SUS. Desta forma, notou-se que a comunicação entre as esferas não foi efetiva todas as vezes, havendo lacunas e demora para a sequência do atendimento das demandas apresentadas pelos usuários, demonstrando deficiências na coordenação do cuidado (Oliveira et al., 2019).

Uma parte considerável dos entrevistados afirmaram procurar primeiro a UBS frente a um novo problema de saúde, tendo, então, a APS como porta de entrada ao serviço de saúde. Entretanto, no geral, grande parte da população ainda utiliza a emergência como admissão única a esse sistema, sendo este um problema enraizado, inclusive, no pensamento popular que associa doença ao hospital, corroborando para a desarticulação entre os níveis de atenção (Oliveira, Veras \& Cordeiro, 2018).

A proximidade da residência à UBS, relatada como principal motivo para que os usuários não trocassem de serviço de saúde, mesmo expondo falhas quanto ao seu funcionamento, foi indicada também como um dos motivos para a não utilização do telefone para comunicação com a equipe. Isso aponta para um aspecto favorável em relação à divisão das áreas por zoneamento do município. Ademais, a maior parte dos entrevistados afirmaram que não apresentavam dificuldades para conseguir atendimento médico, dado discordante de um estudo realizado com homens de Campina Grande, na Paraíba, em que esse item da acessibilidade apresentou escore abaixo do recomendado na maioria das entrevistas (Alves et al., 2020). 
Tomadas em conjunto, os componentes referentes à longitudinalidade foram bem avaliados. A grande maioria dos entrevistados afirmaram se sentir à vontade nas consultas, o que corresponde ao observado em um estudo realizado no Rio Grande do Sul (Kessler et al., 2019). Ademais, a maior parcela dos participantes acredita que os profissionais da Atenção Básica reconhecem os problemas mais importantes em sua vida, sendo que a porcentagem obtida no presente estudo é maior que o observado na pesquisa gaúcha (Kessler et al., 2019). Ainda nessa perspectiva, houve uma frequência predominante de entrevistados que afirmaram que o prontuário estava disponível nas consultas e era utilizado pelo médico. Quando bem escrito e elaborado, o documento fornece informações sobre o paciente e suas consultas anteriores, possibilitando observar a evolução clínica, auxiliando para uma melhor conduta por parte do profissional (Garritano et al., 2020).

Entretanto, quando se considera a importância da longitudinalidade do cuidado, baseada no estabelecimento de um vínculo de confiança entre usuário e equipe de saúde, torna-se preocupante o fato de que mais da metade dos homens afirmaram ter uma grande rotatividade de profissionais da saúde, sendo o achado no presente estudo superior ao obtido no estudo realizado no Rio Grande do Sul (Kessler et al., 2019). Diversos fatores contribuem para que ocorra a rotatividade, como, por exemplo, o valor da remuneração paga ao profissional, a modalidade de contratação, a infraestrutura e a estruturação da rede de saúde da cidade em que se atua (Pierantoni et al., 2015). Deste modo, fica evidente que este processo favorece com que ocorra uma fragilização do vínculo entre as pessoas atendidas e os profissionais da ESF, o que pode repercutir negativamente na longitudinalidade do cuidado.

Quanto aos aconselhamentos contemplados pelo instrumento, apenas a disponibilização de informações sobre a prática de exercícios físicos e a manutenção de uma alimentação saudável receberam uma avaliação positiva. A análise do aconselhamento sobre como parar de fumar constitui uma exceção, já que os dados obtidos podem estar relacionados a uma possível falha do instrumento, uma vez que o mesmo não considera os indivíduos que não são tabagistas e que, $\operatorname{logo}$, não teriam a necessidade de receber conselhos a respeito. Ademais, observou-se a necessidade de incorporar orientações sobre o envelhecimento, uma vez que, apesar da maioria dos entrevistados ser composta por idosos, apenas uma pequena parcela dos participantes afirmou receber esse tipo de aconselhamento. Em uma proposta de modelo assistencial ao idoso, destacou-se a importância de tais informações baseadas em ações educativas, abrangendo doenças predominantes na terceira idade, prática de hábitos de vida saudável, uso correto de medicações, vacinação e seguridade dos ambientes frequentados pelo idoso, o que inclui prevenção de quedas (Oliveira, Veras \& Cordeiro, 2018).

Pode-se destacar ainda que, apesar de um número considerável dos entrevistados afirmar já ter sido diagnosticado com algum problema de saúde mental, a maioria negou ter recebido algum tipo de aconselhamento sobre essa condição. Um estudo realizado na Paraíba, indicou fragilidades envolvidas no manejo da saúde mental na APS, como ausência de grupos terapêuticos voltados para esse público, além da abordagem baseada em renovação de receitas, sem avaliação do progresso do tratamento e até mesmo sem o estabelecimento de diagnóstico que justifique o uso de tais medicações (Silva et al., 2019).

Nas ESF pesquisadas, a orientação familiar encontra-se fragilizada, já que um pequeno número de entrevistados respondeu que a ESF a qual pertencem incluiria a família em reuniões para o manejo de alguma patologia que por ventura viesse a ser apresentada. Os dados obtidos na avaliação deste atributo são concordantes com um estudo realizado em uma região do estado de Goiás em que a maioria dos entrevistados também avaliaram de forma negativa esse atributo (Moreira et al., 2021). É válido ressaltar, ainda, que o apoio familiar durante o tratamento, principalmente de doenças crônicas, pode ser um fator favorável de adesão, uma vez que o núcleo familiar pode colaborar tanto no apoio social quanto na estimulação de práticas voltadas para o autocuidado (Santos et al., 2019).

Por fim, nota-se que há uma falha nos mecanismos de avaliação das unidades, já que grande parcela dos indivíduos estaria participando pela primeira vez por entrevista similar. Estas pesquisas deveriam fazer parte da rotina das equipes, visto 
que a avaliação dos serviços de saúde pela população assistida contribui para direcionar a tomada de decisão de gestores, identificando as principais lacunas e obstáculos existentes no sistema (Protasio et al., 2017; Pucci et al., 2020).

\section{Conclusão}

Verificou-se que a qualidade da Atenção Primária à Saúde de um município mineiro obteve uma avaliação negativa na visão de usuários adultos do sexo masculino assistidos pelas Estratégias de Saúde da Família analisadas pelo estudo. Frente a isso, destaca-se a necessidade da implementação de ações que visem a acessibilidade do homem jovem aos serviços ofertados pela Atenção Básica ao mesmo passo que se tenha ações de políticas públicas voltadas para grupos vulneráveis em saúde idosos, negros, pessoas de baixa escolaridade e baixa renda - que constituíram maioria quantitativa no presente estudo.

Quanto às condições de saúde autorreferidas, observou-se uma boa atuação da ESF no manejo da hipertensão arterial, refletindo em uma melhor orientação da APS quando avaliada por entrevistados hipertensos. Desse modo, tal abordagem pode se constituir um modelo exemplar para outras condições de caráter crônico, como o diabetes.

Por fim, o PCATool versão adulto reduzida se mostrou uma eficaz ferramenta de avaliação da qualidade da APS. Desta forma, pode ser utilizado em outros estudos referentes à saúde do homem, com objetivo de contribuir para o aumento do número de trabalhos sobre essa temática uma vez que há uma pequena quantidade de pesquisas sobre este assunto. Além disso, pode, ainda, ser aplicado para os demais grupos populacionais, a fim de contribuir para que os atributos da Atenção Básica sejam contemplados na integralidade e na diversidade humana.

\section{Referências}

Alves A. N., Coura A. S., França I. S., Magalhães I. M. O., Rocha M. A., \& Araújo R. S. (2020). Acesso de primeiro contato na atenção primária: uma avaliação pela população masculina. Rev Bras Epidemiol, 23: e200072.

Balbino C. M., Silvino Z. R., Santos J. S., Joaquim F. L., Souza C. J., Santos L. M., \& Izu M. (2020). Os motivos que impedem a adesão masculina aos programas de atenção à saúde do homem. Research, Society and Development, 9(7):1-17, e389974230.

Bidinotto D.N., Simonetti J. P., \& Bocchi S. C. (2016). A saúde do homem: doenças crônicas não transmissíveis e vulnerabilidade social. Rev Lat Am Enfermagem, 24 (e2756).

Borges D. B., \& Lacerda J. T. (2018). Ações voltadas ao controle do Diabetes Mellitus na Atenção Básica: proposta de modelo avaliativo. Saúde em debate, 42(116), 162-178.

Brasil. (2009). Ministério da Saúde (BR), Política Nacional de Atenção Integral à Saúde do Homem. Ministério da Saúde.

Brasil. (2010). Manual do instrumento de avaliação da atenção primária à saúde: primary care assessment tool pcatool -Brasil. Ministério da Saúde.

Brasil. (2013). Ministério da Saúde (BR), Diretrizes de atenção à reabilitação da pessoa com acidente vascular cerebral. Ministério da Saúde.

Brasil. (2017). Portaria $n^{\circ}$ 2436, de 21 de setembro de 2017. Aprova a Política Nacional de Atenção Básica, estabelecendo a revisão de diretrizes para a organização da Atenção Básica, no âmbito do Sistema Único de Saúde (SUS).

Brasil. (2018). Ministério da Saúde (BR), e-SUS Atenção Básica: manual de preenchimento das fichas de coleta de dados simplificada: CDS - Versão 3.0. Brasília (DF): Ministério da Saúde.

Dantas R. C., \& Roncalli A. G. (2019). Protocolo para indivíduos hipertensos assistidos na Atenção Básica em Saúde. Cien Saude Colet, 24(1), $295-306$.

Garritano C. R., Junqueira F. H., Lorosa E. F., Fujimoto M. S., \& Martins W. H. A. (2020). Avaliação do prontuário médico de um hospital universitário. Rev Bras Educ Med, 44(1).

Huque R., Nasreen S., Ahmed F., Hicks, J. P., Walley J., Newell J. N., \& Elsey H. (2018). Integrating a diabetes and hypertension case management package within primary health care: a mixed methods feasibility study in Bangladesh. BMC Health Serv Res, 18(811).

Kessler M., Lima S. B., Weiller T. H., Lopes L. F. D., Ferraz L., Eberhardt T. D., Soares R. S. A., \& Trindade L. L. (2019). Longitudinalidade do cuidado na atenção primária: avaliação na perspectiva dos usuários. Acta paulista de enfermagem, 32(2):186-193.

Lages S. R., Silva A. M., Silva D. P., Damas J. M., \& Jesus M. A. (2017). O preconceito racial como determinante social da saúde: a invisibilidade da anemia falciforme. Gerais: Revista Interinstitucional de Psicologia, 10(1), 109-122.

Morais J. D., Ribeiro K. S., \& Paes N. A. (2019). Apoio social e satisfação de hipertensos com a atenção básica: construção de um índice sintético. Saúde em debate, 43(121), 477-488. 
Moreira I. A. V., Resende A. V., Maia L. G., Judice M. G., Mota A. V. S., Ribeiro C. M., Pasieka C. R., \& Silva J. R. M. (2021). Avaliação do acesso ao serviço prestado pela Atenção Primária de Saúde na Região de Saúde Sudoeste I do Estado de Goiás. Research, Society and Development, 10(4), e26910414111.

Moura F. M., \& Vale A. R. (2019). Mercado de trabalho nos pequenos municípios e polarização regional: uma análise sobre Divisa Nova e Alfenas, no Sul/Sudoeste de Minas. Espaço em Revista, 20(2), 1-18.

Neves R. G., Duro S. M., \& Muñiz J., Castro T. R., Facchini L. A., \& Tomasi E. (2018). Estrutura das unidades básicas de saúde para atenção às pessoas com diabetes: Ciclos I e II do Programa Nacional de Melhoria do Acesso e da Qualidade. Cad Saude Publica, 34(4).

Oliveira B. L., Cardoso L. F., Dominice R. O., Corrêa A. A. P., Fonseca A. E. C., Moreira J. P. L., \& Luiz R. R. (2020). A influência da Estratégia Saúde da Família no uso de serviços de saúde por adultos hipertensos no Brasil. Rev Bras Epidemiol, 23 (e200006).

Oliveira C. R., Samico I. C., \& Mendes M. F., Vargas I., \& Vázquez M. L. (2019). Conhecimento e uso de mecanismos para articulação clínica entre níveis em duas redes de atenção à saúde de Pernambuco, Brasil. Cad Saude Publica, 35(4).

Oliveira M. M. (2007). Presença e extensão dos atributos da Atenção Primária à Saúde entre os serviços de Atenção Primária em Porto Alegre: uma análise agregada. Porto Alegre: Universidade Federal do Rio Grande do Sul.

Oliveira M. M., Harzheim E., Riboldi J., \& Duncan B. B. (2013). PCATool-ADULTO-BRASIL: uma versão reduzida Revista brasileira de medicina de família e comunidade, 8(29), 256-263.

Oliveira M. R., Veras R. P., Cordeiro H. A. (2018). A importância da porta de entrada no sistema: o modelo integral de cuidado para o idoso. Physis, 28(4).

Paula C. C., Silva C. B., Tassinari T. T., \& Padoin S. M. M. (2016). Fatores que interferem no acesso de primeiro contato na atenção primária à saúde: revisão integrativa. Revista de pesquisa, cuidado é fundamental, 8(1), 4056-4078.

Pierantoni C. R., Vianna C. M., França T., Magnago C., \& Rodrigues M. P. S. (2015). Rotatividade da força de trabalho médica no Brasil. Saúde em debate, 39(106), 637-647.

Poças K. C., Perillo R. D., Bernal R. T., Maita D. C., \& Duarte E. C. (2019). Primeira escolha para utilização de serviços de saúde pela população adulta do Distrito Federal, 2015: um inquérito de base populacional. Epidemiol Serv Saude, 28 (2).

Portela P. P., Mussi F. C., Gama G. G., \& Santos C. A. S. T. (2016). Fatores associados ao descontrole da pressão arterial em homens. Acta paulista de enfermagem, 29(3), 307-315.

Protasio A. P., Gomes L. B., Machado L. S., \& Valença A. M. G. (2017). Satisfação do usuário da Atenção Básica em Saúde por regiões do Brasil: $1^{\text {o }}$ ciclo de avaliação externa do PMAQ-AB. Cien Saude Colet, 22(6), 1829-1844.

Pucci V. R., Halberstadt B. M. K., Bandeira D., Damaceno A. N., Gomes B. C. F., \& Weiller T. H. (2020). Avaliação dos serviços de Atenção Primária à Saúde: estudo das tendências em teses brasileiras. Research, Society and Development, 9(9):1-28, e402997581.

Queiroz T. S., Rehen T. C., Stival M. M., Funghetto S. S., Lima L. R., Cardoso B. G., \& Santos W. S. (2018). Como homens idosos cuidam de sua própria saúde na atenção básica? Rev. Bras. Enferm., 71(1), 554-561.

Ribeiro C. R., Gomes R., \& Moreira M. C. (2017). Encontros e desencontros entre a saúde do homem, a promoção da paternidade participativa e a saúde sexual e reprodutiva na atenção básica. Physis, 27(1), 41-60.

Salci M. A., Silva D. M., \& Meirelles B.H. (2018). Avaliação no sistema de saúde brasileiro. Ciência, cuidado e saúde, 17(2).

Santos F. G., Mezzavila V. A., Rêgo A. S., Salci M. A., \& Radovanovic C. A. T. (2019). Enfoque familiar e comunitário da Atenção Primária à Saúde a pessoas com hipertensão arterial. Saúde em debate, 43(121), 489-502.

Silva A. N., Silva S. A., Silva A. R., Araújo T. M. E., Rebouças C. B. A., \& Nogueira L. T. (2018). A avaliação da atenção primária a saúde na perspectiva da população masculina. Rev. Bras. Enferm, 71(2), 236-243.

Silva P. M., Costa N. F., Barros D. R., Junior J. A., Silva J. R. L., \& Brito T. S. (2019). Saúde mental na atenção básica: possibilidades e fragilidades do acolhimento. Revista Cuidarte, 10(1).

Sociedade Brasileira de Diabetes. (2019)._Conduta terapêutica no diabetes tipo 2: algoritmo SBD 2019. Posicionamento oficial SBD n 1 / 2019.

Souza Y. V., Gomes R. S., Sá B. V., Mattos R. M. P. R., \& Pimentel D. M. M. (2020). Percepção de pacientes sobre sua relação com médicos. Revista bioética, 28(2), 332-343.

Starfield, B. (2002). Atenção primária: equilíbrio entre necessidades de saúde, serviços e tecnologia. UNESCO, Ministério da Saúde. 